\title{
Normal Fluctuations and the FKG Inequalities
}

\author{
C. M. Newman^ \\ Department of Mathematics, University of Arizona, Tucson, AZ 85721, USA
}

\begin{abstract}
In a translation invariant pure phase of a ferromagnet, finite susceptibility and the FKG inequalities together imply convergence of the block spin scaling limit to the infinite temperature Gaussian fixed point. This result is presented in a rather general probabilistic context and is applicable to infinite cluster density fluctuations in percolation models and to boson field fluctuations in (Euclidean) Yukawa quantum field theory models as well as to magnetization fluctuations in Ising models.
\end{abstract}

\section{Introduction and Results}

We consider a $d$-dimensional cubic lattice of random variables, $\left\{X_{k}: k=\left(k_{1}, \ldots, k_{d}\right) \in \mathbb{Z}^{d}\right\}$, and for each integer $n \geqq 1$ the associated block variables $\left\{X_{k}^{n}: k \in \mathbb{Z}^{d}\right\}$ defined by

$$
X_{k}^{n}=\left(S_{k}^{n}-\left\langle S_{k}^{n}\right\rangle\right) / n^{d / 2},
$$

where $\langle\cdot\rangle$ denotes expectation,

$$
S_{k}^{n}=\sum_{j \in B_{k}^{n}} X_{j}
$$

and $B_{k}^{n}$ is a "block" of side length $n$ located near $n k$ :

$$
\begin{aligned}
B_{k}^{n} & =\left\{j: n k_{l} \leqq j_{l}<n\left(k_{l}+1\right) \quad \text { for } \quad l=1, \ldots, d\right\} \\
& =n k+B_{0}^{n} .
\end{aligned}
$$

We will present a simple natural set of conditions on $\left\{X_{k}\right\}$ (see Theorem 2 below) which insure (in renormalization group terminology [26]) convergence of $\left\{X_{k}^{n}\right\}$ as $n \rightarrow \infty$ to the infinite temperature Gaussian fixed point; i.e., which insure that as $n \rightarrow \infty$,

$$
\left\{X_{k}^{n}: k \in \mathbb{Z}^{d}\right\} \rightarrow\left\{Z_{k}: k \in \mathbb{Z}^{d}\right\}
$$

* $\quad$ Alfred P. Sloan Research Fellow, on leave from Indiana University. Research supported in part by National Science Foundation Grant MCS77-20683 and by the U.S.-Israel Binational Science Foundation 
where the $Z_{k}$ 's are mutually independent normal random variables of mean 0 and some fixed variance $A$, in the sense that for any bounded continuous function $F$ (on $\mathbb{R}^{\mathbb{Z}^{d}}$ ) which only depends on finitely many coordinates,

$$
\lim _{n \rightarrow \infty}\left\langle F\left(\left\{X_{k}^{n}\right\}\right)\right\rangle=\left\langle F\left(\left\{Z_{k}\right\}\right)\right\rangle .
$$

By standard results of probability theory a formulation equivalent to (5) is that

$$
\left\langle\exp \left(i \sum_{k \in \Lambda} r_{k} X_{k}^{n}\right)\right\rangle \rightarrow \exp \left(-\sum_{k \in \Lambda} A r_{k}^{2} / 2\right)
$$

for any finite $\Lambda \subset \mathbb{Z}^{d}$ and any choice of real $r_{k}$ 's.

Two important recent papers concerning convergence to the infinite temperature fixed point are [1] and [11]. In [11] can be found references to a number of papers discussing the relation between the renormalization group approach and the central limit theorem (see also $[7,13,3]$ ), as well as a number of early papers (such as [8]) on the central limit theorem for Ising models. These early papers (see also [12]) typically needed to assume rather strong decrease properties of the correlation functions; however, in situations such as ours where

$$
0 \leqq \operatorname{Cov}\left(X_{j}, X_{k}\right) \equiv\left\langle X_{j} X_{k}\right\rangle-\left\langle X_{j}\right\rangle\left\langle X_{k}\right\rangle=C(k-j)
$$

and where a simple estimation of the variance of $X_{k}^{n}$ (see Lemma 4 in Sect. 2) suggests that the $A$ of (6) is given by

$$
A=\sum_{k \in \mathbb{Z}^{d}} \operatorname{Cov}\left(X_{0}, X_{k}\right)
$$

it seems clear that the "correct" hypothesis is

$$
\sum_{k \in \mathbb{Z}^{d}} \operatorname{Cov}\left(X_{0}, X_{k}\right)<\infty \text {. }
$$

The first result based essentially on this hypothesis was that of Baker and Krinsky [1]; our result should be considered as a natural extension and improvement of theirs. It is an improvement primarily because our hypotheses are considerably weaker and consequently have a much wider range of applicability; for example the results of [1] only apply to Ising models in zero magnetic field above the critical temperature, do not apply with arbitrary single-site distributions, and moreover would not apply to Yukawa or percolation models. The more recent results of Iagolnitzer and Souillard [11] are based on the Lee-Yang theorem and are thus of a somewhat different nature than ours or those of [1]. From one point of view the methods of [11] are more general than ours since they should apply to any model in which appropriate information about zeros of the partition function can be obtained (and thus could be used to study energy fluctuations for example) whereas our methods require the validity of the FKG inequalities. On the other hand, even in the standard Ising model their results are valid only away from a phase transition whereas ours should apply to a pure magnetized phase at zero magnetic field below the critical temperature.

In this section of the paper, we present our main results. Theorem 1 gives a new inequality concerning multivariate characteristic functions for random variables satisfying the FKG inequalities. Theorem 2 is our main result concerning 
convergence to the infinite temperature fixed point. Following Theorem 2, we discuss various models to which it can be applied and then consider the situation when $\operatorname{Cov}\left(X_{0}, X_{k}\right)$ is "barely" divergent. All proofs are presented in Sect. 2 of the paper; the key ingredient in the proof of Theorem 2 is the inequality of Theorem 1.

A real function $F$ on $\mathbb{R}^{m}$ will be called increasing if

$x_{l} \leqq y_{l} \quad$ for all $l$ implies

$F\left(x_{1}, \ldots, x_{m}\right) \leqq F\left(y_{1}, \ldots, y_{m}\right)$.

A finite set of random variables $\left\{X_{1}, \ldots, X_{m}\right\}$ will be said to satisfy the FKG inequalities if for any increasing $F, G$,

$$
\operatorname{Cov}\left(F\left(X_{1}, \ldots, X_{m}\right), G\left(X_{1}, \ldots, X_{m}\right)\right) \geqq 0,
$$

assuming the two random variables in (10) have finite variance; i.e., assuming

$$
\left\langle\left[F\left(X_{1}, \ldots, X_{m}\right)\right]^{2}\right\rangle<\infty
$$

and similarly for $G$. An infinite set of random variables will be said to satisfy the FKG inequalities if every finite subset satisfies them.

The FKG inequalities originated in work on percolation models [10], were extended to spin-1/2 Ising models in [6], and have been generalized in many ways since (see [14] and the references given there). They have also been discussed in the Statistics literature $[17,4]$. Our use of the FKG inequalities is strongly motivated by the work of Lebowitz in [15] and by subsequent related results in [23] and [25, Sect. III-0].

Theorem 1. Suppose $Y_{1}, \ldots, Y_{m}$ have finite variance and satisfy the FKG inequalities; then for any real $r_{1}, \ldots, r_{m}$,

$$
\begin{aligned}
& \left|\left\langle\exp \left(i \sum_{l=1}^{m} r_{l} Y_{l}\right)\right\rangle-\prod_{l=1}^{m}\left\langle\exp \left(i r_{l} Y_{l}\right)\right\rangle\right| \\
& \quad \leqq \frac{1}{2} \sum_{l \neq n} \sum_{l}\left|r_{l}\right|\left|r_{n}\right| \operatorname{Cov}\left(Y_{l}, Y_{n}\right) .
\end{aligned}
$$

Remark. (11) is based on the interesting Lemma 3 presented below in Sect. 2. A multivariate version of Lemma 3 can be obtained which shows that for smooth complex valued $F, G$ on $\mathbb{R}^{m}$ and $\bar{F}=F\left(Y_{1}, \ldots, Y_{m}\right), \bar{G}=G\left(Y_{1}, \ldots, Y_{m}\right)$,

$$
|\operatorname{Cov}(\bar{F}, \bar{G})| \leqq C \sum_{l=1}^{m} \sum_{n=1}^{m}\left\|\partial F / \partial y_{l}\right\|_{\infty} \cdot\left\|\partial G / \partial y_{n}\right\|_{\infty} \cdot \operatorname{Cov}\left(Y_{l}, Y_{n}\right)
$$

where $\|\cdot\|_{\infty}$ denotes the sup norm on $\mathbb{R}^{m}$ and $C$ is a universal constant (no larger than $3 \sqrt{2}$ ). Inequality (12) is not needed for the proof of Theorem 2 , but it can be used, for example, to show [assuming hypotheses A)-C) of Theorem 2] that ergodicity of the $j$-shift $\left(0 \neq j \in \mathbb{Z}^{d}\right)$ is equivalent to :

$$
\lim _{n \rightarrow \infty} \frac{1}{n} \sum_{l=1}^{n} \operatorname{Cov}\left(X_{0}, X_{k+l j}\right)=0 \text { for all } k \in \mathbb{Z}^{d} .
$$

Although (12) seems to require the full strength of the FKG inequalities, our proof of Theorem 1 shows that (11) remains valid if one only assumes that for any choice 
of $r_{l}, s_{l} \geqq 0$ with $r_{l} s_{l}=0$,

$$
\begin{aligned}
& P\left(\sum r_{l} X_{l}>x, \sum s_{l} Y_{l}>y\right) \\
& \quad \geqq P\left(\sum r_{l} X_{l}>x\right) \cdot P\left(\sum s_{l} Y_{l}>y\right) \text { for all } x, y \in \mathbb{R} .
\end{aligned}
$$

As a consequence, hypothesis C) of Theorem 2 can be weakened.

Theorem 2. Suppose $\left\{X_{k}: k \in \mathbb{Z}^{d}\right\}$ satisfies the following:

A) Finite Variance: $\left\langle X_{k}^{2}\right\rangle<\infty$ for all $k \in \mathbb{Z}^{d}$.

B) Translation Invariance: for all $m$ and for all $j, k_{1}, \ldots, k_{m} \in \mathbb{Z}^{d},\left(X_{k_{1}}, \ldots, X_{k_{m}}\right)$ has the same distribution as $\left(X_{j+k_{1}}, \ldots, X_{j+k_{m}}\right)$.

C) FKG Inequalities.

D) Finite "Susceptibility":

$$
A \equiv \sum_{k \in \mathbb{Z}^{d}} \operatorname{Cov}\left(X_{0}, X_{k}\right)<\infty .
$$

Then the block variables $\left\{X_{k}^{n}: k \in \mathbb{Z}^{d}\right\}$, as defined in (1)-(3) converge to independent mean zero Gaussian random variables of variance A (the infinite temperature fixed point) in the sense of (6) (weak convergence of finite dimensional distributions).

Remark. When $d=1$, the convergence of (6) can be strengthened to yield an "invariance principle" so that an analogue of $(S)$ is valid for a larger class of $F$ "s; this result will be presented in [22]. For general $d$, one may define the generalized random field on $\mathbb{R}^{d}$,

$$
\psi_{n}(\mathbf{u})=\sum_{j \in \mathbb{Z}^{d}}\left(X_{j}-\left\langle X_{j}\right\rangle\right) \delta(\mathbf{u}-j / n) / n^{d / 2},
$$

and using standard arguments show that as a consequence of Theorem $2, \psi_{n}$ converges to $d$-dimensional Gaussian white noise of parameter $\mathrm{A}$ in the sense that (say for real functions $f$ in the Schwartz space $\mathscr{S}\left(\mathbb{R}^{d}\right)$ )

$$
\left\langle\exp \left(i \int_{\mathbb{R}^{d}} \psi_{n}(\mathbf{u}) f(\mathbf{u}) d \mathbf{u}\right)\right\rangle \rightarrow \exp \left(-\int_{\mathbb{R}^{d}} A(f(\mathbf{u}))^{2} d \mathbf{u} / 2\right)
$$

Theorem 2 is sufficiently general that it is applicable to a wide range of models. We proceed to briefly discuss three examples.

\section{General Ising Models}

In such a model with Hamiltonian, $-\sum_{j, k} J(j-k) \sigma_{j} \sigma_{k}-h \sum_{j} \sigma_{j}$, and single spin distribution $d \varrho\left(\sigma_{j}\right)$ (see [24, Sect. VIII-3] for basic definitions and terminology), we may take $X_{k}$ to be the spin variable $\sigma_{k}$. The FKG inequalities will be valid for any $\varrho$ and $h$, providing $J(k) \geqq 0$ for all $k$ [9]. Theorem 2 should in principle be applicable to any translation invariant pure phase which is not at a critical point. It is also possible to apply Theorem 2 to a critical phase by looking at magnetization fluctuations in a lower dimensional slice; for example, at the critical point of the standard three dimensional Ising model where $\operatorname{Cov}\left(\sigma_{0}, \sigma_{k}\right)$ is believed to behave asymptotically like $\|k\|^{-(1+\eta)}$ for some small positive $\eta(\|\cdot\|$ denoting Euclidean distance), one could apply Theorem 2 to the one-dimensional slice $\left\{X_{j}: X_{j}=\sigma_{(j, 0,0)}\right.$, $\left.j \in \mathbb{Z}^{1}\right\}$. 


\section{Percolation Models}

If in a general Ising model of the above type one has the single spin distribution $d \varrho\left(\sigma_{j}\right)=p \delta\left(\sigma_{j}-1\right)+(1-p) \delta\left(\sigma_{j}\right)$ with $p \in(0,1)$ and one defines $X_{j}$ to be 1 (respectively 0 ) if $j$ belongs (respectively doesn't belong) to an infinite cluster of occupied sites (i.e., $X_{j}$ is 1 if there is an infinite sequence $j=j_{0}, j_{1}, j_{2}, \ldots$ of distinct sites in $\mathbb{Z}^{d}$ with $j_{l+1}$ a nearest neighbor of $j_{l}$ and $\sigma_{j_{l}}=1$ for each $l$ ), then the FKG inequalities are valid both in the independent case $(J \equiv 0)[10]$ and in the correlated case $(J \neq 0)$ [6]. Theorem 2 in this context concerns normal fluctuations of "infinite cluster density" and is discussed in more detail in [21].

\section{Yukawa Models}

It has recently been shown that the FKG inequalities are valid for the boson field $\phi$ in a Euclidean Yukawa quantum field theory (with the fermion field "integrated out") [2]. We may take $X_{k}$ to be $\phi$ integrated over a unit cube centered at $k$. In order to discuss extensions of Theorem 2 when (13) is invalid, we replace the block variables given in (1) by

$$
Y_{k}^{n}=\left(S_{k}^{n}-\left\langle S_{k}^{n}\right\rangle\right) /\left(\operatorname{Var}\left(S_{k}^{n}\right)\right)^{1 / 2} .
$$

The weakest condition which would insure that for $k \neq j, \operatorname{Cov}\left(Y_{k}^{n}, Y_{j}^{n}\right) \rightarrow 0$ as $n \rightarrow \infty$ is that the function

$$
K(R) \equiv \sum_{\|k\| !} \operatorname{Cov}\left(X_{0}, X_{k}\right)
$$

(where $\|\cdot\|$ again denotes Euclidean distance in $\mathbb{R}^{d}$ ) be slowly varying as $R \rightarrow \infty$; i.e.,

$$
\lim _{R \rightarrow \infty} \frac{K(\lambda R)}{K(R)}=1 \quad \text { for any } \quad \lambda>0
$$

(see [5, Sect. VIII-8] for various properties of slowly varying functions). This condition allows a logarithmic divergence in $\sum_{k} \operatorname{Cov}\left(X_{0}, X_{k}\right)$ as one would have if for example, $\operatorname{Cov}\left(X_{0}, X_{k}\right) \sim$ Const $\cdot\|k\|^{-d}$. Note that asymptotically the denominator in (14) can be replaced by $n^{d / 2}(K(n))^{1 / 2}$ since it can be shown that $\operatorname{Var}\left(S_{k}^{n}\right) /\left[n^{d} K(n)\right] \rightarrow 1$.

Conjecture. If $\left\{X_{k}\right\}$ satisfies hypotheses A)-C) of Theorem 2 and condition (16) is valid, then (6) will be valid (for any finite $\Lambda$ and real $r_{k}$ 's) with $X_{k}^{n}$ replaced by $Y_{k}^{n}$ and A replaced by 1.

Remark. We have been able to prove a weaker version of this conjecture in which the following additional hypothesis is assumed:

$$
\lim _{\lambda \rightarrow \infty}\left(\sup _{n}\left\langle\left(Y_{0}^{n}\right)^{2} 1_{\left\{\left|Y_{O}^{n}\right|>\lambda\right\}}\right\rangle\right)=0,
$$

where $1_{B}$ denotes the indicator function of the event $B$. Since a uniform bound on $\left\langle\left|Y_{0}^{n}\right|^{p}\right\rangle$ for $p>2$ would imply (17), it follows that (17) would be valid if, for example, $\left\langle X_{j}\right\rangle=0$ and

$$
\left\langle\left(S_{0}^{n}\right)^{4}\right\rangle \leqq 3\left\langle\left(S_{0}^{n}\right)^{2}\right\rangle^{2}
$$


since then $\left\langle\left(Y_{0}^{n}\right)^{4}\right\rangle \leqq 3\left\langle\left(Y_{0}^{n}\right)^{2}\right\rangle^{2}=3\left[\operatorname{Var}\left(Y_{0}^{n}\right)\right]^{2}=3$. In certain Ising models, (18) is valid as a consequence of either the Lebowitz inequality [16], the Lee-Yang theorem [18], or the GHS inequality [19]. It is interesting to note that if one takes $X_{k}$ as the local energy density in the standard two-dimensional Ising model at its critical point, then $K(R)$ has exactly the type of logarithmic divergence allowed by (16); in this case however the FKG inequalities do not apply (for a discussion of the block variable limit in this case, see the remark following Corollary 2.10 in [20]). A situation where both (18) and the FKG inequalities are valid and where $K(R)$ should be slowly varying with a logarithmic singularity, can be manufactured by taking a two dimensional slice (as described in the discussion on general Ising models above) out of the critical phase of the standard four dimensional Ising model.

\section{Proofs}

A pair of random variables, $X, Y$, are said to be positive quadrant dependent [17] if

$$
\begin{aligned}
H_{X, Y}(x, y) & \equiv P(X \leqq x, Y \leqq y)-P(X \leqq x) \cdot P(Y \leqq y) \\
& \equiv P(X>x, Y>y)-P(X>x) P(Y>y) \geqq 0 \text { for all } x, y \in \mathbb{R} .
\end{aligned}
$$

It is easily seen that this condition is valid if $\{X, Y\}$ satisfies the FKG inequalities. In [17] it was shown that such an $X$ and $Y$ must be independent if they are uncorrelated, by using the following identity (for $X, Y$ with finite variance):

$$
\operatorname{Cov}(X, Y)=\int_{-\infty}^{\infty} \int_{-\infty}^{\infty} H_{X, Y}(x, y) d x d y
$$

The following lemma generalizes this argument.

Lemma 3. Suppose $X, Y$ are positive quadrant dependent with finite variance and $f, g$ are $C^{1}$ complex valued functions on $\mathbb{R}^{1}$ with $f^{\prime}, g^{\prime}$ bounded. Then

$$
|\operatorname{Cov}(f(X), g(Y))| \leqq\left\|f^{\prime}\right\|_{\infty} \cdot\left\|g^{\prime}\right\|_{\infty} \operatorname{Cov}(X, Y),
$$

where $\|\cdot\|_{\infty}$ denotes the sup norm on $\mathbb{R}^{1}$; in particular, for any real $r, s$

$$
\left|\left\langle e^{i r X+i s Y}\right\rangle-\left\langle e^{i r X}\right\rangle\left\langle e^{i s Y}\right\rangle\right| \leqq|r||s| \operatorname{Cov}(X, Y) .
$$

Proof. (19) can be easily generalized to yield

$$
\operatorname{Cov}(f(X), g(Y))=\int_{-\infty}^{\infty} \int_{-\infty}^{\infty} f^{\prime}(x) g^{\prime}(y) H_{X, Y}(x, y) d x d y
$$

thus by the positivity of $H_{X, Y}$ and by (19),

$$
\begin{aligned}
|\operatorname{Cov}(f(X), g(Y))| & \leqq \int_{-\infty}^{\infty} \int_{-\infty}^{\infty}\left|f^{\prime} \| g^{\prime}\right| H_{X, Y} d x d y \\
& \leqq\left\|f^{\prime}\right\|_{\infty}\left\|g^{\prime}\right\|_{\infty} \operatorname{Cov}(X, Y),
\end{aligned}
$$

as desired. 
Proof of Theorem 1. We proceed by induction on $m$. The result is true for $m=1$ trivially and for $m=2$ by Lemma 3; we suppose it is true for $m \leqq M$. For $m=M+1$ we may (by rearranging the indices if necessary) assume that for some $\varepsilon= \pm 1$, $\delta= \pm 1$, and $m^{\prime} \in\{1, \ldots, M\}, \varepsilon r_{l} \geqq 0$ for $1 \leqq l \leqq m^{\prime}$ while $\delta r_{l} \geqq 0$ for $m^{\prime}+1 \leqq l \leqq M+1$. We then define

$$
X=\sum_{l=1}^{m^{\prime}} \varepsilon r_{l} Y_{l}, \quad Y=\sum_{l=m^{\prime}+1}^{M+1} \delta r_{l} Y_{l}
$$

and note that since $\left\{Y \ldots, Y_{m}\right\}$ satisfy the FKG inequalities so do $X$ and $Y$ (because increasing functions of increasing functions are increasing). Now since $\sum_{1}^{m} r_{l} Y_{l}=\varepsilon X+\delta Y$, we have by (21) and the induction hypothesis that the left hand side of (11) is bounded by

$$
\begin{aligned}
& \left|\left\langle e^{i(\varepsilon X+\delta Y)}\right\rangle-\left\langle e^{i \varepsilon X}\right\rangle\left\langle e^{i \delta Y}\right\rangle\right| \\
& +\left|\left\langle e^{i \varepsilon X}\right\rangle\left\langle e^{i \delta Y}\right\rangle-\left\langle e^{i \varepsilon X}\right\rangle \prod_{m^{\prime}+1}^{M+1}\left\langle\exp \left(i r_{l} Y_{l}\right)\right\rangle\right| \\
& +\left|\left\langle e^{i \varepsilon X}\right\rangle \prod_{m^{\prime}+1}^{M+1}\left\langle\exp \left(i r_{l} Y_{l}\right)\right\rangle-\left(\prod_{1}^{m^{\prime}}\left\langle\exp \left(i r_{l} Y_{l}\right)\right\rangle\right) \prod_{m^{\prime}+1}^{M+1}\left\langle\exp \left(i r_{l} Y_{l}\right)\right\rangle\right| \\
& \leqq \\
& \quad|\varepsilon||\delta| \operatorname{Cov}(X, Y)+\left|\left\langle e^{i \delta Y}\right\rangle-\prod_{m^{\prime}+1}^{M+1}\left\langle\exp \left(i r_{l} Y_{l}\right)\right\rangle\right| \\
& \quad+\left|\left\langle e^{i \varepsilon X}\right\rangle-\prod_{1}^{m^{\prime}}\left\langle\exp \left(i r_{l} Y_{l}\right)\right\rangle\right| \\
& \leqq \\
& \quad \operatorname{Cov}\left(\sum_{1}^{m^{\prime}} \varepsilon r_{l} Y_{l^{\prime}} \sum_{m^{\prime}+1}^{M+1} \delta r_{n} Y_{n}\right)+\frac{1}{2} \sum_{\substack{l \neq n \\
m^{\prime}+1}}^{M+1}\left|r_{l}\right|\left|r_{n}\right| \operatorname{Cov}\left(Y_{l}, Y_{n}\right) \\
& \quad+\frac{1}{2} \sum_{l \neq n}^{m^{\prime}}\left|r_{l}\left\|r_{n}\left|\operatorname{Cov}\left(Y_{l}, Y_{n}\right)=\frac{1}{2} \sum_{l \neq n}\right| r_{l}\right\| r_{n}\right| \operatorname{Cov}\left(Y_{l}, Y_{n}\right)
\end{aligned}
$$

which completes the proof.

The proof of Theorem 2 is based upon the following lemma and the simple fact that

$$
X_{0}^{m l}=l^{-d / 2} \sum_{j \in B_{0}^{l}} X_{j}^{m} .
$$

Lemma 4. Suppose $\left\{X_{k}: k \in \mathbb{Z}^{d}\right\}$ have finite variance and $0 \leqq \operatorname{Cov}\left(X_{j}, X_{k}\right)=C(k-j)$ for all $j, k$ with

$$
A \equiv \sum_{k \in \mathbb{Z}^{d}} C(k)<\infty ;
$$

then for any $k, j \in \mathbb{Z}^{d}$,

$$
\begin{aligned}
& \lim _{n \rightarrow \infty} \operatorname{Var}\left(X_{k}^{n}\right)=A, \\
& \lim _{n \rightarrow \infty}\left\langle\left(X_{0}^{m_{n}}-X_{0}^{n}\right)^{2}\right\rangle=0 \text { if } \lim _{n \rightarrow \infty} m_{n} / n=1,
\end{aligned}
$$


and

$$
\lim _{n \rightarrow \infty} \operatorname{Cov}\left(X_{k}^{n}, X_{j}^{n}\right)=0 \quad \text { if } \quad k \neq j .
$$

Proof. We first note that

$$
\begin{aligned}
\operatorname{Var}\left(X_{k}^{n}\right) & =n^{-d} \sum_{j \in B_{k}^{n}} \sum_{k \in B_{k}^{n}} C(k-j) \\
& \leqq n^{-d} \sum_{j \in B_{k}^{n}} \sum_{k \in \mathbb{Z}^{d}} C(k-j)=A .
\end{aligned}
$$

For $0<\varepsilon<1 / 2$, we define

$$
B_{0}^{n}(\varepsilon)=\left\{j: \varepsilon n<j_{l}<(1-\varepsilon) n \text { for } l=1, \ldots, d\right\}
$$

so that for $j \in B_{0}^{n}(\varepsilon)$ and $k \notin B_{0}^{n},\|k-j\|>\varepsilon n$; thus

$$
\begin{aligned}
\operatorname{Var}\left(X_{k}^{n}\right) & =\operatorname{Var}\left(X_{0}^{n}\right) \geqq n^{-d} \sum_{j \in B_{0}^{n}(\varepsilon)} \sum_{k \in B_{0}^{n}} C(k-j) \\
& \geqq n^{-d} \sum_{j \in B_{0}^{n}(\varepsilon)} \sum_{\|k-j\| \leqq \varepsilon n} C(k-j)=\frac{\left|B_{0}^{n}(\varepsilon)\right|}{n^{d}}\left(\sum_{|| k|| \leqq \varepsilon n} C(k)\right),
\end{aligned}
$$

where $|B|$ denotes the cardinality of $B$. If we choose $\varepsilon=\varepsilon_{n}$ so that $\varepsilon_{n} \rightarrow 0$ while $\varepsilon_{n} n \rightarrow \infty$, then the right hand side of (31) tends to $A$; this together with (29) yields (26). Next we note that

$$
\begin{aligned}
\left\langle\left(X_{0}^{m_{n}}-X_{0}^{n}\right)^{2}\right\rangle & =\operatorname{Var}\left(X_{0}^{m_{n}}\right)+\operatorname{Var}\left(X_{0}^{n}\right)-2\left(m_{n} n\right)^{-d / 2} \operatorname{Cov}\left(S_{0}^{m_{n}}, S_{0}^{n}\right) \\
& \leqq \begin{cases}\operatorname{Var}\left(X_{0}^{m_{n}}\right)+\operatorname{Var}\left(X_{0}^{n}\right)-2\left(n / m_{n}\right)^{d / 2} \operatorname{Var}\left(X_{0}^{n}\right) & \text { if } . m_{n} \geqq n \\
\operatorname{Var}\left(X_{0}^{m_{n}}\right)+\operatorname{Var}\left(X_{0}^{n}\right)-2\left(m_{n} / n\right)^{d / 2} \operatorname{Var}\left(X_{0}^{m_{n}}\right), & \text { if } \quad m_{n} \leqq n .\end{cases}
\end{aligned}
$$

Now since $m_{n} / n \rightarrow 1$, we have by (26) that the right hand side of (32) tends to zero which yields (27). In order to obtain (28), we note that since $\operatorname{Cov}\left(X_{k}^{n}, X_{j}^{n}\right)=\operatorname{Cov}\left(X_{0}^{n}, X_{k^{\prime}}^{n}\right)$ (where $k^{\prime}= \pm(k-j)$ with the sign chosen so that for some $\left.l, k_{l}^{\prime}>0\right)$, it suffices by (27) to prove that

$$
\lim _{n \rightarrow \infty} \operatorname{Cov}\left(X_{0}^{m_{n}}, X_{k^{\prime}}^{n}\right)=0,
$$

where we choose $m_{n}$ so that $m_{n} / n \rightarrow 1$ while $n-m_{n} \rightarrow+\infty$. Now in this case we have

$$
\begin{aligned}
\operatorname{Cov}\left(X_{0}^{m_{n}}, X_{k^{\prime}}^{n}\right) & =m_{n}^{-d / 2} n^{-d / 2} \sum_{j \in B_{0}^{m_{n}}} \sum_{k \in B_{k^{\prime}}^{n}} C(k-j) \\
& \leqq\left(m_{n} / n\right)^{d / 2}\left(m_{n}\right)^{-d} \sum_{j \in B_{0}^{m_{n}}} \sum_{\|k-j\| \geqq n-m_{n}} C(k-j) \\
& =\left(m_{n} / n\right)^{d / 2} \sum_{\|k\| \geqq n-m_{n}} C(k) \rightarrow 0
\end{aligned}
$$

which completes the proof of Lemma 4.

Proof of Theorem 2. We first note that it suffices to prove (6) with $\Lambda=\{0\}$ since then by translation invariance (6) would be valid for $\Lambda=\{k\}$, and consequently, since for arbitrary $\Lambda,\left\{X_{k}^{n}: k \in \Lambda\right\}$ satisfies the FKG inequalities, it would follow by 
(11) (with $\left.\left\{Y_{l}\right\}=\left\{X_{k}^{n}\right\}\right)$ and (28) that

$$
\begin{aligned}
& \lim _{n \rightarrow \infty}\left|\left\langle\exp \left(i \sum_{k \in \Lambda} r_{k} X_{k}^{n}\right)\right\rangle-\exp \left(-\sum_{k \in \Lambda} A r_{k}^{2} / 2\right)\right| \\
& =\lim _{n \rightarrow \infty}\left|\left\langle\exp \left(i \sum_{k \in \Lambda} r_{k} X_{k}^{n}\right)\right\rangle-\prod_{k \in \Lambda}\left\langle\exp \left(i r_{k} X_{k}^{n}\right)\right\rangle\right| \\
& \quad \leqq \lim _{n \rightarrow \infty} \frac{1}{2} \sum_{\substack{k, j \in \Lambda \\
k \neq j}}\left|r_{k}\right|\left|r_{j}\right| \operatorname{Cov}\left(X_{k}^{n}, X_{j}^{n}\right)=0,
\end{aligned}
$$

which would yield (6) for general finite $\Lambda$ as desired. We next note, as a consequence of (27), that for fixed $m=1,2, \ldots$

$$
\begin{aligned}
& \left|\left\langle\exp \left(i r X_{0}^{n}\right)\right\rangle-\left\langle\exp \left(i r X_{0}^{m[n / m]}\right)\right\rangle\right| \\
& \quad \leqq\left\langle\left|\exp \left(i r\left(X_{0}^{m[n / m]}-X_{0}^{n}\right)\right)-1\right|\right\rangle \\
& \quad \leqq\left\langle\left|X_{0}^{m[n / m]}-X_{0}^{n}\right|\right\rangle \leqq\left\langle\left(X_{0}^{m[n / m]}-X_{0}^{n}\right)^{2}\right\rangle^{1 / 2} \rightarrow 0,
\end{aligned}
$$

where $[\cdot]$ denotes the usual greatest integer function. Next, by $(24)$ (with $l=[n / m]$ ) and (11) (with $\left\{Y_{l}\right\}=\left\{X_{j}^{m}\right\}$ and $r_{l}=r l^{-d / 2}$ ) and (26) (with $k=0$ ), we have as $l \rightarrow \infty$,

$$
\begin{aligned}
& \left|\left\langle\exp \left(i r X_{0}^{m l}\right)\right\rangle-\left\langle\exp \left(i r l^{-d / 2} X_{0}^{m}\right)\right\rangle^{l^{d}}\right|<\frac{1}{2} \sum_{\substack{j, k \in B_{0}^{l} \\
j \neq k}} r^{2} l^{-d} \operatorname{Cov}\left(X_{j}^{m}, X_{k}^{m}\right) \\
& =\frac{r^{2}}{2}\left(\operatorname{Cov}\left(X_{0}^{m l}, X_{0}^{m l}\right)-l^{-d} \sum_{j \in B_{0}^{l}} \operatorname{Cov}\left(X_{j}^{m}, X_{j}^{m}\right)\right) \\
& =\frac{r^{2}}{2}\left(\operatorname{Var}\left(X_{0}^{m l}\right)-\operatorname{Var}\left(X_{0}^{m}\right)\right) \rightarrow \frac{r^{2}}{2}\left(A-A_{m}\right)
\end{aligned}
$$

where we have used in two places the fact that (by translation invariance) $X_{j}^{m}$ and $X_{0}^{m}$ are identically distributed and have defined $A_{m} \equiv \operatorname{Var}\left(X_{0}^{m}\right)$. Finally, as in the proof of the standard central limit theorem (for i.i.d. random variables with finite variance) [5, Sect. XV-5], we have as $l \rightarrow \infty$ for fixed $m$

$$
\begin{aligned}
& \left\langle\exp \left(i r l^{-d / 2} X_{0}^{m}\right)\right\rangle^{l^{d}} \\
& =\left(1-\frac{r^{2}}{2} l^{-d} A_{m}+o\left(l^{-d}\right)\right)^{l^{d}} \rightarrow \exp \left(-A_{m} r^{2} / 2\right) .
\end{aligned}
$$

Now, by combining (35), (36), and (37), we obtain

$$
\begin{aligned}
& \limsup _{n \rightarrow \infty}\left|\left\langle\exp \left(\operatorname{ir} X_{0}^{n}\right)\right\rangle-\exp \left(-A r^{2} / 2\right)\right| \\
& \quad \leqq \frac{r^{2}}{2}\left(A-A_{m}\right)+\left(\left(\exp \left(-A_{m} r^{2} / 2\right)\right)-\exp \left(-A r^{2} / 2\right)\right)
\end{aligned}
$$

since this is true for any $m$ and since by (26) $A_{m} \rightarrow A$ as $m \rightarrow \infty$, we have that

$$
\lim _{n \rightarrow \infty}\left\langle\exp \left(\operatorname{ir} X_{0}^{n}\right)\right\rangle=\exp \left(-A r^{2} / 2\right)
$$

which is just (6) with $\Lambda=\{0\}$ as desired. This completes the proof. 


\section{References}

1. Baker, G.A., Jr., Krinsky, S.: Renormalization group structure for translationally invariant ferromagnets. J. Math. Phys. 18, 590-607 (1977)

2. Battle, G.A., Rosen, L.: The FKG inequality for the Yukawa ${ }_{2}$ quantum field theory. Preprint, Univ. of British Columbia (1979)

3. Ellis, R.S., Newman, C.M.: Fluctuationes in Curie-Weiss exemplis. In: Mathematical problems in theoretical physics. Dell' Antonio, G. Doplicher, S., Jona-Lasinio, G. (eds.). Berlin, Heidelberg, New York: Springer 1978

4. Esary, J., Proschan, F., Walkup, D.: Association of random variables with applications. Ann. Math. Stat. 38, 1466-1474 (1967)

5. Feller, W.: An introduction to probability theory and its applications, Vol. II, 2nd ed. New York: Wiley 1966

6. Fortuin, C., Kastelyn, P., Ginibre, J.: Correlation inequalities on some partially ordered sets. Commun. Math. Phys. 22, 89-103 (1971)

7. Gallavotti, G., Jona-Lasinio, G.: Limit theorems for multidimensional Markov processes. Commun. Math. Phys. 41, 301-307 (1975)

8. Gallavotti, G., Martin-Löf, A.: Block-spin distributions for short range attractive Ising models. Nuovo Cimento 25B, 425-441 (1975)

9. Guerra, F., Rosen, L., Simon, B.: The $P(\phi)_{2}$ Euclidean quantum field theory as classical statistical mechanics. Ann. Math. 101, 111-259 (1975)

10. Harris, T.E.: A lower bound for the critical probability in a certain percolation process. Proc. Camb. Phil. Soc. 59, 13-20 (1960)

11. Iagolnitzer, D., Souillard, B.: Lee-Yang theory and normal fluctuations. Phys. Rev. B19, $1515-1518$ (1979)

12. Iagolnitzer, D., Souillard, B.: Random fields and limit theorems. To appear in the proceedings of the Estergom 1979 Conference on "Random fields: rigorous results in statistical mechanics and quantum field theory"

13. Jona-Lasinio, G.: Probabalistic approach to critical behavior. In: New developments in quantum field theory and statistical mechanics. Lévy, M., Mitter, P. (eds.). New York: Plenum 1977

14. Kemperman, J.H.B.: On the FKG-inequalities for measures on a partially ordered space. Indag. Math. 39, 313-331 (1977)

15. Lebowitz, J.: Bounds on the correlations and analyticity properties of ferromagnetic Ising spin systems. Commun. Math. Phys. 28, 313-321 (1972)

16. Lebowitz, J.: GHS and other inequalities. Commun. Math. Phys. 35, 87-92 (1974)

17. Lehman, E.L.: Some concepts of dependence. Ann. Math. Stat. 37, 1137-1153 (1966)

18. Newman, C.M.: Inequalities for Ising models and field theories which obey the Lee-Yang theorem. Commun. Math. Phys. 41, 1-9 (1975)

19. Newman, C.M.: Moment inequalities for ferromagnetic Gibbs distributions. J. Math. Phys. 16, 1956-1959 (1975)

20. Newman, C.M.: Critical point inequalities and scaling limits. Commun. Math. Phys. 66, 181-196 (1979)

21. Newman, C.M., Schulman, L.S. : Infinite clusters in percolation models (in preparation)

22. Newman, C.M., Wright, A.L.: An invariance principle for certain dependent sequences (in preparation)

23. Simon, B.: Correlation inequalities and the mass gap in $P(\phi)_{2}$. I. Domination by the two point function. Commun. Math. Phys. 31, 127-136 (1973)

24. Simon, B.: The $P(\phi)_{2}$ Euclidean (quantum) field theory. Princeton: Princeton University Press 1974

25. Wells, D.R.: Some moment inequalities and a result on multivariate unimodality. Indiana University $\mathrm{Ph}$. D. Thesis, 1977

26. Wilson, K.G., Fischer, M.E. : Critical exponents in 3.99 dimensions. Phys. Rev. Lett. 28, 240-243 (1972)

Communicated by E. Lieb

Received December 12, 1979 